\title{
Through-space intervalence charge transfer in cobalt-based Metal-organic framework: an experimental and theoretical study
}

\author{
A. Nath, S. Tanty, V. S. Reddy, S. Mandal \\ School of Chemistry, IISER Thiruvananthapuram, Kerala, India \\ iakashdeepnath16@iisertvm.ac.in
}

Metal-Organic Frameworks (MOFs) are a class of crystalline organic-inorganic hybrid materials derived from metal nodes and organic linkers, that exhibit features like high surface area, well-defined pore, tunable structures and their properties [1]. Use of redoxactive metal nodes or organic linkers, stable radical based ligands can introduce a special feature like conductivity, electrocatalyst, electrochromic behavior in MOFs apart from their conventional uses such as gas storage, gas separation, etc. This idea is impeded mainly due to the insulating nature of organic linkers and the instability of the framework to the redox process. This hindered the study of electroactive MOFs until the last decade. Recent advancement in this field has directed a surge of interest in understanding their mechanism of charge transfer. MOFs are a unique platform to investigate the charge transfer mechanism where the corresponding metal ions or organic linkers are well defined in a highly crystalline rigid system. Charge transfer is directed by either the through-space or through-bond approach [2]. The through-bond mixed-valance charge transfer has been well explored whereas, through-space intervalency in MOF is rare [3].

We have synthesized a new Cobalt (II) based metal-organic framework using redox-active organic linker, N,N'-di(4-pyridyl)thiazolo[5,4-d]thiazole (DPTTZ). The framework exhibits through-space intervalence charge transfer (IVCT) arise from cofacially arranged DPTTZ linkers (Figure 1). The IVCT is elucidated computationally using time-dependent density functional theory (TD-DFT) methods. The computational study also exploits the distance-dependent through-space intervalence charge transfer (IVCT) in this system.

Here, I will present experimental observation of through-space intervalence charge transfer (IVCT) using redox-active organic linkers in the metal-organic framework and its computational understanding using TD-DFT. This interrogation of charge transfer mechanism and electrical conductivity in MOF provides a better understanding of conducting materials.

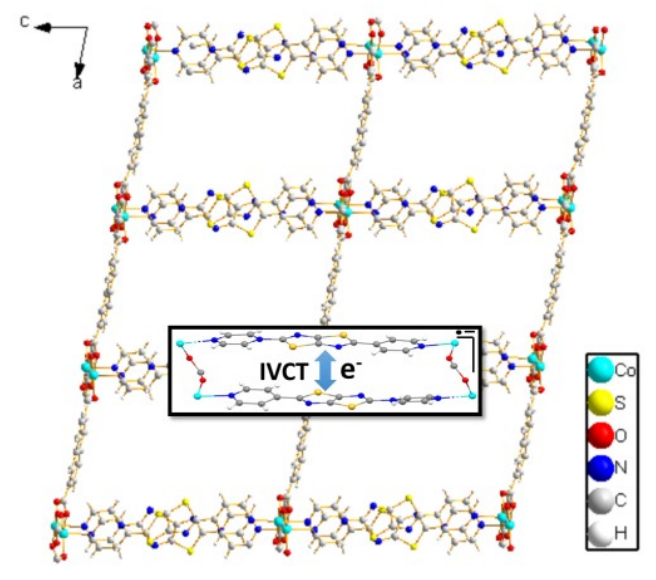

Figure 1. Crystal structure showing cofacial arrangement of DPTTZ ligands and through-space IVCT (inset).

[1] Furukawa, H., Cordova, K. E., O'Keeffe, M., Yaghi, O. M. Science 2013, 341, 1230444.

[2] Sun, L.; Campbell, M. G.; Dincă, M. Angew. Chem. Int. Ed. 2016, 55, 3566.

[3] Hua, C. et al. J. Am. Chem. Soc. 2018, 140, 6622.

[4] Nath, A. et al. (Manuscript under preparation)

Keywords: Metal-organic framework; charge transfer; conductivity; redox-active ligand; density functional theory Akashdeep Nath acknowledges the institute fund of IISER Thiruvananthapuram for his financial support. 